\title{
Tumor de glomus yugulotimpánico, a propósito de un caso
}

\author{
(Jugulotympanic glomus tumor, case presentation)
}

Jazmín Reyes-Carmona, ${ }^{1}$ David Salazar-Olmedo, Alejandro Vargas-Román²

\section{Resumen}

Los tumores de glomus yugulo-timpánico 0 paragangliomas son originados de células de la cresta neural y representan un número bajo de los tumores de cabeza y cuello $(0,5 \%)$. Su crecimiento lento y síntomas progresivos provocan que se retarde el diagnóstico. Conocer la clínica que frecuentemente cursa con pérdida auditiva unilateral, tinnitus pulsátil y afectación de algún par craneal, puede ayudar a sospechar el diagnóstico de glomus. Se mencionan diferentes tipos de métodos diagnósticos, así como nuevas opciones de tratamiento. Dada la localización y su importante vascularización, hay casos en los que la cirugía abierta es muy riesgosa y se buscan procedimientos terapéuticos que disminuyan las complicaciones y morbilidades en estos pacientes, tanto como procedimientos endovasculares. En el artículo se discute el caso de una femenina de 31 años con clínica de inicio insidioso con hipoacusia izquierda progresiva, tinnitus pulsátil izquierdo y disfonía. En 2013, se realiza resonancia magnética nuclear donde se observa proceso infiltrativo tumoral sugestivo de paraganglioma. Se hace embolización endovascular con onyx de glomus yugulotimpánico en cuatro ocasiones, con seguimiento cada 6 meses por no ser candidata a otro tipo de tratamiento, debido al tamaño y localización de la masa y su condición no resecable.

Descriptores: paraganglioma, glomo yugular, glomo timpánico.

\section{Abstract}

Jugulo-tympanic glomus tumors or paragangliomas originate from neural crest cells and represent a low number of head and neck tumors $(0.5 \%)$. Its slow growth and progressive symptoms cause the diagnosis to be delayed.

Afiliación de los autores: "Universidad de Iberoamérica, ${ }^{2}$ Servicio de Neurocirugía Hospital "Dr. Rafael Ángel Calderón Guardia", San José, Costa Rica. Conflictos de interés: no existen.

凶jazzreyesc@gmail.com

ISSN 0001-6012/2020/62/1/43-46

Acta Médica Costarricense, () 2020

Colegio de Médicos y Cirujanos

de Costa Rica
The knowledge of the clinic that frequently involves unilateral hearing loss, pulsatile tinnitus and cranial nerve involvement can help to suspect the diagnosis. Different types of diagnostic methods are helpfun, as well as new treatment options have been proposed. Given the location and its important vascularization, there are cases in which open surgery is highly risky and alternative therapeutic procedures trying to reduce complications and morbidities in these patients need to be considered, as well as endovascular procedures. This article discusses the case of a 31-year-old female with an insidious onset of progressive left hearing loss, left pulsatile tinnitus and dysphonia. In 2013, nuclear magnetic resonance imaging was performed and a tumor infiltrative process suggesting paraganglioma was observed. Endovascular embolization was performed with onyx of jugulotympanic glomus on four occasions, with follow-up every 6 months, due to the size and location of the mass and its unresectable condition the tumor was considered non surgically resectable.

Keywords: glomus jugulare, glomus tympanicum.

Fecha recibido: 20 de marzo 2019

Fecha aprobado: 14 de noviembre 2019

El glomus yugulotimpánico pertenece a una clasificación de tumores también llamados paragangliomas o quemodectomas, los cuales son tumores benignos muy vascularizados. ${ }^{1,2}$ Las células son originarias de estructuras quimiorreceptoras de la cresta neural embrionaria, las cuales se pueden encontrar en el cuerpo carotídeo, nervio vago, oído medio, nervio timpánico, rama del nervio glosofaríngeo (nervio de Jacobson), rama auricular del nervio vago (nervio de Arnold) y foramen yugular. 1,3-5 Estas estructuras son quimiorreceptores que ayudan a regular la presión de oxígeno, y existen en mayor tamaño en el recién nacido, ya que en el período embrionario son importantes en la producción de catecolaminas, pero van degenerando poco a poco en la niñez, y su función es sustituida por la glándula suprarrenal.6,7 Se encuentran ligadas al sistema nervioso autónomo, y responden a los cambios en el oxígeno, pH y dióxido de carbono. ${ }^{8}$

La incidencia de dicho tumor varía, en los Estados Unidos la serie más grande es de 99 casos publicados a lo largo de 31 años, aproximadamente 1 caso en 1,3 millones. ${ }^{1,2}$ Se estima que representa entre el 0,5\% y el 0,6\% de la incidencia de tumores de cabeza y cuello. $5,6,8$ Los glomus más comunes son los que afectan el cuerpo carotídeo seguido de los yugulares. ${ }^{9}$ Afecta normalmente a adultos entre los 20 y 60 años, siendo 
más frecuente en mujeres, en relación 4:1.2 Se ha observado que su incidencia es mayor en pacientes con hipoxia crónica o habitantes de zonas con mayor altura sobre el nivel del mar. ${ }^{10}$ Alrededor de un 10 \% tendrá agregación familiar, y suele ser múltiple hasta en un 35 a un $50 \%$ de los casos. ${ }^{3}$

Se observa a una paciente femenina portadora de un tumor de cabeza y cuello de poca incidencia, el cual demuestra una forma ideal de describir la clínica con la que se presenta la mayoría de los glomus yugulares y timpánicos, notándose progresivamente el daño de las estructuras adyacentes con sus consecuentes síntomas. Con normalidad los tumores de glomus yugulotimpánicos son resecados mediante cirugía abierta; sin embargo, este caso representa una excepción, ya que por su tamaño y localización, no fue candidato a cirugía, de manera que se repasan las opciones terapéuticas conservadoras, como cirugía estereotáctica y métodos endovasculares para manejo de dicha afección.

\section{Presentación del caso}

Femenina de 31 años, en control desde 2013 en Otorrinolaringología (ORL) del Hospital Calderón Guardia, por historia de inicio de hipoacusia izquierda progresiva posterior a trauma timpánico dos años previos, que posteriormente asoció tinnitus pulsátil izquierdo, disfonía y disfagia lógica. Al llegar al servicio de ORL, se realiza otoscopía, la cual evidencia una lesión pulsátil violácea que llena todo el conducto auditivo externo izquierdo distalmente, sin otorrea. Se efectúa

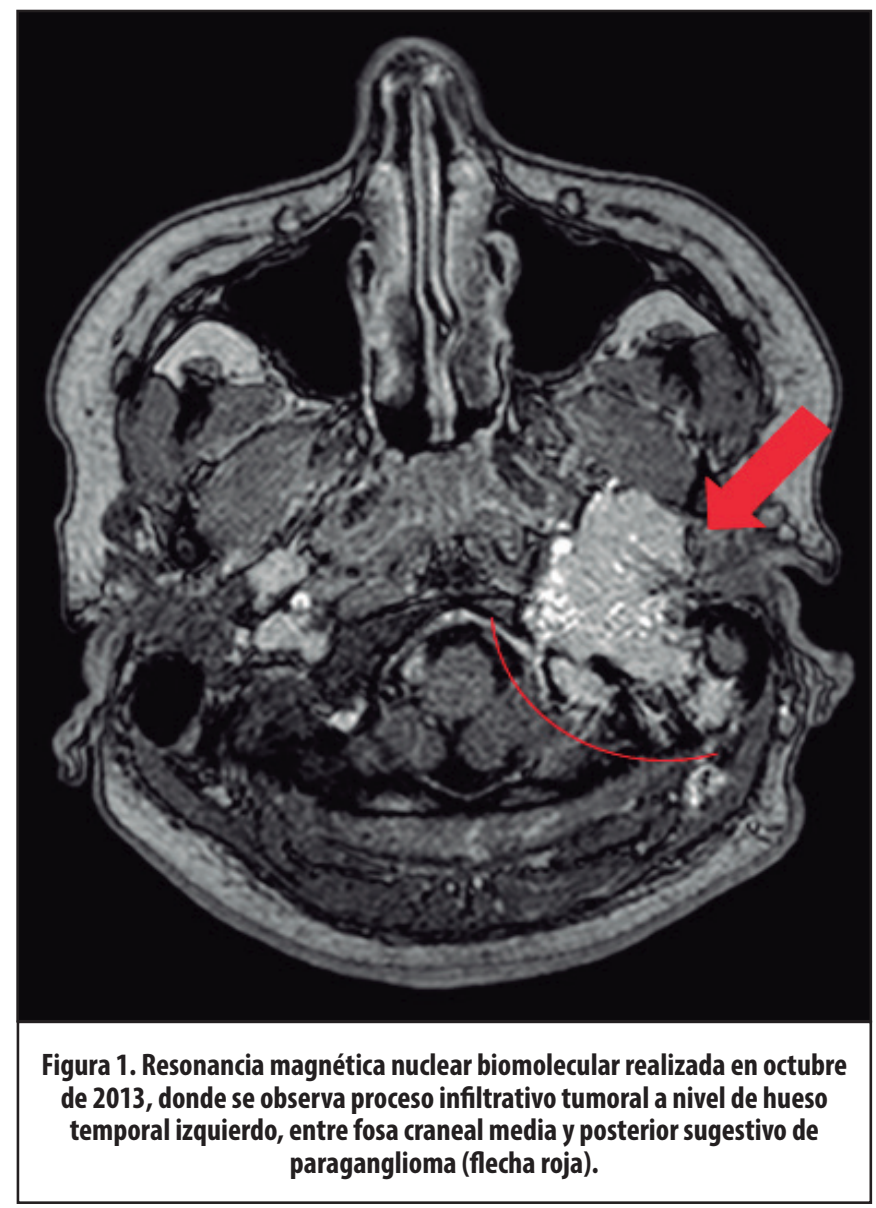

tomografía axial computada en octubre de 2013, donde se describe que impresiona erosión del tegmento timpánico y masa que llena oído medio y parte del oído externo. Resonancia magnética nuclear (RMN) demuestra un proceso infiltrativo tumoral, localizado en la fosa yugular izquierda que abarcaba el hipotímpano, proyectándose en el conducto auditivo externo del lado izquierdo, inferiormente extendido al espacio carotídeo izquierdo y en el área superior infiltrando la duramadre, que recubría el seno sigmoideo y el seno transverso del lado izquierdo, con un componente de tejidos blandos retromastoideo izquierdo; la lesión refuerza tras la administración de contraste y presenta muchos pedículos vasculares intratumorales, y produce ligero efecto de masa, dado por la compresión del parénquima cerebeloso adyacente.

Posterior a la RMN se establece una impresión diagnóstica de meningioma versus paraganglioma (Figura 1). En noviembre de 2013 se lleva paciente a hemodinamia por el Servicio de Neurocirugía para embolización neuroendovascular con onyx de glomus yugulotimpánico Fisch D2 (Cuadro 1), y de nuevo se realiza embolización residual una semana después con onyx, donde se encontró persistencia de ramas maxilar interna y occipitales. En dicha ocasión se logra embolizar el 100 \% del aporte arterial. La paciente fue egresada en ese momento en buenas condiciones, persistiendo con hipoacusia, y ameritó gastrostomía endoscópica percutánea y uso de sonda para alimentación durante 5 meses, por persistencia de disfagia; por lo demás, con sus capacidades conservadas. En 2015 se realizó una segunda embolización neuroendovascular de la lesión, por nuevo crecimiento, y en 2017, durante la cita de control, la paciente refiere cuello abotagado y cambios en el tono de la voz, por lo que se repite la RMN, que demostró persistencia de glomus yugulotimpánico. En esta ocasión se desarrolla sesión multidisciplinaria con ORL y neurocirugía para valorar posibilidad de resección de lesión con Gamma knife, lo cual se solicitó a 2 hospitales extranjeros (Miami y Colombia), y ambos rechazaron el caso por el tamaño de la masa.

A inicios de 2019 la paciente continuó control con nuevo crecimiento de masa, por lo que es llevada a arteriografía cerebral diagnóstica, con colocación de angioseal y diagnóstico postquirúrgico de glomusyugular izquierdo del todo embolizado a nivel de base craneal, con angiogénesis en ramas proximales de carótida externa izquierda. Se observa pequeña rama de la arteria cerebral posterior izquierda, la cual alimenta residuo

\begin{tabular}{|c|c|}
\hline \multicolumn{2}{|r|}{$\begin{array}{c}\text { Cuadro 1. Clasificación, según etapas de glomus } \\
\text { yugulo-timpánico (Fisch) }\end{array}$} \\
\hline Tipo & Descripción \\
\hline A & Tumor limitado a promontorio \\
\hline B & $\begin{array}{l}\text { Tumor confinado a oído medio, hipotímpano y } \\
\text { mastoides }\end{array}$ \\
\hline C & $\begin{array}{l}\text { Tumor que erosiona bulbo yugular y foramen } \\
\text { caratídeo; compromiso infralaberíntico }\end{array}$ \\
\hline D1 & Invasión intracraneal $<2 \mathrm{~cm}$ \\
\hline D2 & Invasión intracraneal > $2 \mathrm{~cm}$ \\
\hline
\end{tabular}


Tumor de glomus yugulotimpánico / Reyes-Carmona et al

lesional, sin crecimiento en los últimos 3 años. Se da de alta en Neurocirugía; continúa control en ORL cada año.

\section{Discusión}

Desde el punto de vista clínico, los tumores de glomus yugulotimpánico son de crecimiento lento, unilaterales, y como cualquier otra lesión ocupante de espacio, sus síntomas se asocian a las diferentes estructuras que comprimen. La presentación habitual consiste en pérdida auditiva unilateral, tinnitus pulsátil y afectación de algún par craneal dependiendo de su localización: si están ubicados a nivel de fosa craneal media afectarán los pares craneales V y VI; si están ubicados a nivel de la fosa craneal posterior afectarán los pares craneales V, VI, VIII, IX y XII. ${ }^{2}$ Si el tumor afecta el foramen yugular puede dar clínica de síndrome de agujero rasgado posterior, presentando síntomas como: acúfenos pulsátil, vértigo, hipoacusia, disfagia, disfonía y disnea. ${ }^{11}$ En algunos casos puede observarse, por medio de otoscopía, una masa vascular. ${ }^{3}$

En el caso presentado, tenemos una masa de crecimiento progresivo, ya que desde los síntomas iniciales hasta el compromiso de estructuras adyacentes, pasaron 3 años, y entonces presentó los síntomas característicos de pérdida auditiva unilateral, dada posiblemente por obstrucción del canal auditivo, ya que en las resonancias no se menciona compromiso del VIII par craneal. La masa se lograba ver por medio de otoscopía simple y sus síntomas posteriores son atribuidos al compromiso de pares craneales bajos, disfagia por afectación del IX par craneal y disfonía por afectación del X par craneal, cuyo nervio laríngeo recurrente se encarga de inervar las cuerdas vocales. Las secuelas por afectación de estructuras adyacentes fueron controladas, la hipoacusia persistió, sin embargo, la disfagia llegó a resolverse después de un tiempo.

Los tipos de glomus yugulotimpánicos dependen de su tamaño y localización y se establecen en la escala de Fisch. ${ }^{4}$ En este caso tenemos una masa con gran compromiso intracraneal, que comprende fosa yugular, hipotímpano, espacio carotídeo y la duramadre del seno sigmoides, lo cual corresponde a una clasificación de Fish D2, por existir más de $2 \mathrm{~cm}$ de invasión intracraneal.

El estudio imagenológico es de vital importancia para el diagnóstico y para valorar las opciones terapéuticas. La RMN suele ser superior a la tomografía axial computarizada, especialmente en paragangliomas pequeños. ${ }^{12}$ En el caso mostrado ambos tipos de estudios se realizaron en varias ocasiones, para diagnóstico y seguimiento de la lesión. Se puede utilizar RMN con angiografía en procura de visualizar las arterias de alimentación de los paragangliomas, ya que usualmente estos son muy vascularizados; además, pueden observarse signos indirectos que sugieren el diagnóstico de glomus yugulotimpánico, por ejemplo, la compresión del bulbo yugular. ${ }^{12}$ La arteriografía es el estudio más específico y sensible y se utiliza para confirmar el diagnóstico, valorar la afectación funcional de los grandes vasos y conocer la irrigación tumoral, con el fin de predecir posibles complicaciones neurológicas postratamiento y valorar la posibilidad de embolización prequirúrgica, la cual ha demostrado disminuir el sangrado intraoperatorio, el tiempo quirúrgico y el riesgo operatorio., ${ }^{2,13}$.

En el caso comentado no existió posibilidad de resección quirúrgica por el tamaño de la masa y el compromiso de estructuras importantes, por lo cual la arteriografía fue utilizada como forma diagnóstica y curativa; se realizaron embolizaciones con Onyx para reducir el tamaño de la masa en cuatro ocasiones, y seguimiento a la paciente por la posibilidad de nueva vascularización. Se valoraron todas las opciones potencialmente curativas, como el manejo con Gamma Knife en hospitales extranjeros, sin embargo, el caso fue rechazado en dos ocasiones por las dimensiones de la lesión, de manera que el manejo definitivo debió ser con embolizaciones para reducción de la masa.

Se ha definido la cirugía como tratamiento de elección siempre y cuando sean tumores de pequeño tamaño que puedan ser resecados de forma completa, pero, ya existen otras modalidades terapéuticas para glomus, las cuales incluyen la radioterapia, radiocirugía y cirugía estereotáctica (Gamma Knife), por lo cual surge controversia al definir el mejor método para tratar dichos tumores. ${ }^{2,14}$. En un metaanálisis, la radiocirugía demostró curación y disminución de complicaciones quirúrgicas, y en algunos casos se sugiere como tratamiento inicial. ${ }^{15}$ Las opciones no invasivas se recomiendan en pacientes ancianos, individuos con un estado general deficiente, un diagnóstico de tumor avanzado, en quienes rechazan una cirugía o en un tumor no resecable. Se concluye que se debe individualizar para definir el mejor procedimiento terapéutico. ${ }^{14}$

\section{Referencias}

1. Gandía M, Kusak M, Martínez N, Gutiérrez S, Rey G, Martínez R. Jugulotympanic paragangliomas treated with Gamma Knife radiosurgery: a single-center review of 58 cases. J Neurosurg. 2014; 121: 1158-1165.

2. Gamarra E, Layana D. Tumor de glomus yugular, presentación de un caso clínico y revisión de la literatura/ Glomus juglare tumor, case study and bibliograpic reference. Rev medicina (Guayaquil) 2005; 11: 153-157.

3. Pacheco M, Rodríguez M, López A, Canul L, Martínez C, Sánchez D. Expresión de las sintasas de óxido nítrico en tumores glómicos de cabeza y cuello. Rev Sanid Milit Mex. 2006; 60: 369-378.

4. Nazar G, Cabezas L, Godoy J, Goycoolea M, Selman J, Ortiz A, et al. Paragangliomas de cabeza y cuello. Rev. Otorrinolaringol. Cir. Cabeza cuello. 2005; 65: 203-214.

5. Kollert M, Minovi A, Draf W, Bockmuhl U. Cervical paragangliomas tumor control and long-term functional results after surgery. Skull base-interrd ap. 2006; 16.

6. Cáceres H, Silva S, Amarilla R, Laconich D, Sosa K. Tumor de glomus carotídeo. Rev. Cir. Parag. 2014; 38: 35-37.

7. Aguirre H, Toranzo J, Colunga R, Esquivel O. Glomus del cuerpo carotídeo (paraganglioma) Rev. ADM. 2009; 65: 24-27.

8. Ladino L, Delgado J, López A, Arango J. Paraganglioma yugular, reporte de caso. Neurol arg. 2013; 5:114-116.

9. Rodríguez M, Pérez E, Ysa A, Bardón F, Vela P, del Campo A, et al. Paraganglioma del nervio vago. Angiología. 2006; 58:151-155.

10. Ferbeyre L, Cruz P, Cuevas I, Vázquez J. Quemodectomas de la región cervicofacial. Rev. cubana med. 2003; 42:46-51. 
11. Vázquez V, Saynes F, Hernández G. Síndrome de agujero rasgado posterior Casuística y manejo. An. Otorrinolaringol Mex. 2004; 47: 4-8.

12. Van den Berg R, Schepers A, T. de Bruine F, Liauw L, Mertens B, Van der Mey $A$, et al. The value of MR angiography techniques in the detection of head and neck paragangliomas. Eur J Radiol. 2004; 52: 240-245.

13. Pérez A, Saura P, García J, de las Heras J. Diagnóstico y control evolutivo de los paragangliomas de cabeza y cuello. Interés de la angiografía y procedimientos neurorradiológicos intervencionistas. Acta otorrinolaingo Esp. 2009; 60:53-67.

\section{Ejemplo: \\ Articulo de revista:}

- Zalaquett S. Canulación arterial axilar para circulación extracorpórea. Rev med Chile. 2003;131:390-396.

- Lohse N, Hansen ABE, Pedersen G, Kronborg G, Gerstoff J, Sorensen HT, et al. Survival of persons with and without HIV infection in Denmark. Ann Intern Med. 2007;146:87-95.

Libro:Ramírez J. Cirugía cardiaca.2da ed. Bogotá: Editorial Científica,1994.

\section{Capitulo de libro:}

- Piel G. ¿Qué es un artículo científico? En: Day R, ed. Cómo escribir y publicar trabajos científicos. Washington: OMS, 1990:8-14.

- Freedman M Inherited forms of bone marrow failure. In: Hoffman R, ed. Hematology: Basic principles and practice. 4th ed Pennsylvania: Churchill Livingstone, 2005:234-238.

\section{Cita electrónica:}

- Aguilar M. Anestesia para cirugía laparoscópica. 17 páginas. Recuperado el 12 de febrero de 2007. En: http://www.monografias.com

Cuadros. Autoexplicativos, numerados consecutivamente en el texto con números arábigos, y cada uno presentado en forma individual en una hoja aparte, con el número y el título y cualquier nota explicativa en la parte inferior, todo a doble espacio. No usar el anglicismo tabla.

Figuras. Deben ser autoexplicativas. Traer el título en el pie de figura, junto con la explicación detallada de la misma. Las figuras, fotos e ilustraciones deben numerarse consecutivamente con números arábigos, de acuerdo con su primera mención en el texto. Si se aportan imágenes, deben venir en presentación de alta calidad, con buena definición. Se sugiere presentar un archivo en Excel con la información y la figura. Debe brindarse suficiente información, en el pie de figura, para interpretar las mismas sin necesidad de referirse al texto.

\section{Revisiones}

El Comité Editorial solicitará la mayoría de las revisiones a expertos en el área y evaluará artículos de revisión presentados sin solicitud expresa, sobre tópicos de interés médico actual, siempre y cuando incluyan un autor con experiencia clínica o de investigación en el área en la que versa el artículo y brinden información novedosa. El número de autores debe limitarse a tres.

\section{Opinión}

Comunicación de temas de actualidad de la medicina sobre avances científicos, enseñanza, políticas y servicios de salud, aspectos bioéticos y otros de interés médico general. Los artículos de opinión deben limitarse a 3000 palabras y pueden incluir 2 figuras o cuadros.

\section{Comunicaciones breves}

Se consideran solo trabajos originales que sean concisos en su versión definitiva. Estos manuscritos no deberán exceder las 3000 palabras y no incluirán más de 2 cuadros y 2 figuras.

\section{Cartas al editor}

Las concernientes principalmente a artículos publicados en AMC serán consideradas para publicación. Deberán promover discusión científica en temas médicos y dirigirse al Comité Editorial, indicando que son
14. Ramos A, Borkoski S, Pérez D, Lisner I, Arnesto A, Cenjor C, et al. Glomus tumors of temporal bone origin. Study of 17 cases. Acta Otorrinolaringol Esp. 2007; 58: 358-361.

15. Gus Z, Batra S, imb C, Li G, Sughrue M, Redmond K, et al. Radiosurgery of glomus jugular tumors: a meta-analysis. Int J Radiat Oncol Biol Phys. 2011; 81:497-502.

para considerarse como correspondencia y no como artículo original. Pueden contener una figura o cuadro y no más de 500 palabras y 5 referencias. Deberían limitarse a un máximo de 3 autores. Los editores se reservan el derecho de editar cartas con el fin de abreviar o clasificar su contenido.

Se publicarán como cartas al editor los casos clínicos de gran valor que suponen un aporte importante en el conocimiento de la fisiopatología, clínica, tratamiento u otros aspectos de la patología, o que brinden material o información de relevancia para una investigación futura. El número de autores se debe limitar a 4, pero debe incluirse a los directamente involucrados en el caso. El texto máximo para una carta al editor es de 3 páginas a doble espacio, con un límite de 30 líneas por página; máximo 3 figuras o cuadros.

\section{Editoriales}

De preferencia, los editoriales se relacionan con artículos publicados en el mismo número de la revista, o en números recientes o bien cualquier otro tema de relevancia para el grupo médico nacional. Deben limitarse a 1500-1800 palabras.

\section{Presentación del manuscrito}

Los manuscritos deben ser presentados en letra Calibri tamaño 11, una columna, a doble espacio, en tamaño carta, con todas las hojas numeradas de manera consecutiva (incluyendo figuras y cuadros), en forma digital y con una copia impresa. Los cuadros y figuras (con su pie) deben estar cada uno en una hoja.

Los autores deben entregar junto con el manuscrito la "lista de chequeo para autores", la transferencia de derechos, la responsabilidad de participación y la declaración de financiamiento. El manuscrito se entrega en la oficina del Comité Editorial de AMC, en el edificio del Colegio de Médicos y Cirujanos. Se puede enviar al correo electrónico actamedica@medicos.cr; el trabajo solo se puede dar por recibido luego de que se remita un correo de respuesta confirmando recepción y cumplimiento de lo solicitado.

Documentos por adjuntar con el manuscrito: se debe presentar una carta que garantice cada uno de los siguientes puntos; para facilidad de los autores, se recomienda el texto por incluir:

A. Asignación de derechos: adjuntar una carta en los siguientes términos "En consideración a la revista y edición del manuscrito presentado, los autores firmantes, en forma conjunta y unánime, transferimos y asignamos todo derecho, título o interés envueltos, incluyendo cualquier y todo derecho de autor en cualquier tipo de forma y medio, al Colegio de Médicos y Cirujanos de Costa Rica. De no publicarse el trabajo en AMC, estos derechos serán liberados.".

B. Responsabilidad de participación: adjuntar una carta en los siguientes términos: "Yo, el autor firmante, hago constar que he participado suficientemente en el contenido intelectual, el análisis de información (si se aplica), y la escritura del manuscrito, para tomar responsabilidad pública por él. He revisado la versión final del manuscrito y considero que representa trabajo válido y apruebo su publicación. Como autor de este artículo, hago constar que nada del material en él incluido ha sido publicado antes, está incluido en otro manuscrito o está siendo considerado para publicación en otro lugar. También hago constar que este artículo no ha sido aceptado para publicación en otro lugar y que yo no he asignado ningún derecho o interés a ninguna tercera persona. Si los editores de AMC requieren la información original en la que este manuscrito se basa, la entregaré para revisión." Junto al nombre de cada autor, se debe especificar en qué partes del trabajo 